\title{
The Extended TOPSIS Method for Multi-criteria Decision Making Based on Hesitant Heterogeneous Information
}

\author{
Xiaolu Zhang \\ School of Economics and Management, \\ Southeast University \\ Nanjing, China \\ Xiaolu_jy@163.com
}

\author{
Zeshui Xu \\ Business School, Sichuan University, \\ Chengdu, China \\ Xuzeshui@263.net
}

\begin{abstract}
In the real world there often exist some decision situations with high degree of uncertainty where the decision makers hesitate among several values to provide their assessments. In such cases, the decision makers usually employ the hesitant fuzzy sets (HFSs) to express their assessments in the quantitative settings and the hesitant fuzzy linguistic term sets (HFLTSs) in the qualitative ones. This paper analyzes a hesitant heterogeneous multi-criteria decision making problem involved both HFSs and HFLTSs. To handle this sort of decision problems, an extended TOPSIS (Technique for Order Preference by Similarity to Ideal Solution) is proposed. In the proposed method the separations to the ideal solution and negative ideal solution under each criterion are calculated by using different distance measures, respectively. Then the separations under each criterion are normalized in order to ensure the compatibility of all criteria. Afterwards, the weight separations are derived, and the optimal alternative which is closest to the ideal solution and remotest from negative ideal solution is also identified. At length, an example is used to illustrate the proposed approach.
\end{abstract}

Keywords- Hesitant fuzzy sets; hesitant fuzzy linguistic term sets; multi-criteria decision making; TOPSIS

\section{INTRODUCTION}

Multiple criteria decision making (MCDM) is to make an optimal choice that has the highest degree of satisfaction from a set of all feasible alternatives characterized with multiple competing criteria. In the real decision process, these competing criteria in MCDM problems are usually of different nature, which may be qualitative and quantitative. According to the different nature of criteria and the decision maker (DM)'s knowledge area, the assessments provided by the DM may be taken different formats such as real numbers, intervals and linguistic variables, etc. In general, MCDM problems with multiple formats of information are called the heterogeneous MCDM problems. This sort of MCDM problems is very complex and interesting in applications of decision making theory. Many useful and valuable methods have been proposed to solve such MCDM problems [1] [4] [5] [6]. For example, Herrera et al. [4] proposed a method that converts all heterogeneous information into the 2-tuple linguistic information for solving the heterogeneous MCDM problems. Li et al. [6] developed a systematical approach that computes the distances to the positive ideal solution as well as negative ideal solution for each criterion and obtains the multi-attribute ranking index.
However, in the real world there often exist some decision situations with high degree of uncertainty where the DMs hesitate among several values to provide their assessments. When the decision criteria of MCDM problems are quite quantitative because of their nature, the hesitant fuzzy sets (HFSs) proposed by Torra [9] are usually used to manage this situation; while the decision criteria are quite qualitative, the hesitant fuzzy linguistic term sets (HFLTSs) introduced by Rodriguez et al. [7] are employed to capture the corresponding cases. Similar to the heterogeneous MCDM problems, the MCDM problems with multiple hesitant formats of information, such as HFSs and HFLTSs, are called the hesitant heterogeneous MCDM problems. For such situations, the previous methods cannot be used to manage them. To this end, Rodriguez et al. [8] proposed an approach that unifies the heterogeneous information in a linguistic domain by means of the 2-tuple linguistic representation [3] to manage this sort of MCDM problems. In their approach all the heterogeneous information is converted into the 2-tuple linguistic representation by using different transformation functions. However, these transformation processes are very complex and may loss much original information.

To preserve more original information, this study employ the main structure of TOPSIS (Technique for Order Preference by Similarity to Ideal Solution) to proposed a generalization TOPSIS method for solving the hesitant heterogeneous MCDM problems. The proposed method computes the distances to the ideal solution and negative ideal solution under each criterion by using different distance measures, and determines the optimal alternative which is closest to the positive ideal solution and remotest from negative ideal solution. The structure of this paper is organized as follows: Section 2 briefly reviews some concepts of HFSs, HFLTSs and hesitant trapezoidal fuzzy numbers (HTrFNs). Section 3 presents a hesitant heterogeneous MCDM problem and proposes an extended TOPSIS method for solving such a MCDM problem. Section 4 employs a supplier selection example to demonstrate the implementation process of the proposed method. Section 5 presents our conclusions.

\section{PRELIMINARIES}

This section presents some basic concepts of HFSs, HFLTSs and HTrFNs which will be useful in the subsequent sections. 
In [9], Torra introduced a concept of HFS which permits the membership degree of an element to a set to be represented as several possible values between 0 and 1 . The biggest advantage of the HFS is that it can express the hesitancy of human beings efficiently, especially when two or more sources of vagueness appear simultaneously.

Definition 2.1 [9]. Let $X$ be a reference set, a HFS $A$ on $X$ is defined in terms of a function $h_{A}(x)$ when applied to $X$ returns a subset of $[0,1]$.

To be easily understood, Xia and Xu [10] expressed the HFS by a mathematical symbol:

$$
A=\left\{<x, h_{A}(x)>\mid x \in X\right\}
$$

where $h_{A}(x)$ is a set of some different values in $[0,1]$, representing the possible membership degrees of the element $x \in X$ to $A$. For convenience, they called $h_{A}(x)$ a hesitant fuzzy element (HFE) denoted by $h=\left\{\gamma^{f} \mid f=1,2 \cdots ; \# h\right\}$ (\#h is the number of all elements in $h$ ).

Assumption 2.1. Two assumptions are made (see [2], [11], [13], [16-18]): (1) All possible values of the HFE $h$ are arranged in a decreasing order, and thus $\gamma^{f}$ is the $f^{\text {th }}$ largest value in $h$. (2) In order to have a correct comparison, the two corresponding HFEs should have the same length. For two HFEs $h_{1}$ and $h_{2}$, if there are fewer values in $h_{1}$ than in $h_{2}$, an extension of $h_{1}$ should be considered pessimistically by repeating its minimum value until it has the same length with $h_{2}$.

Drawing on the well-known Hamming and Euclidean distance measures, $\mathrm{Xu}$ and $\mathrm{Xia}$ [12] defined two hesitant fuzzy distance measures for HFEs:

Definition 2.2. For any two HFEs $h_{i}=\left\{\gamma_{i}^{f} \mid f=1,2, \cdots, \# h_{i}\right\} \quad(i=1,2)$ with $\# h=\# h_{1}=\# h_{2}$, the hesitant fuzzy Hamming and Euclidean distances between them can be defined, respectively, as follows:

$$
d_{1}\left(h_{1}, h_{2}\right)=\frac{1}{\# h} \sum_{f=1}^{\# h}\left|\gamma_{1}^{f}-\gamma_{2}^{f}\right|
$$

and

$$
d_{2}\left(h_{1}, h_{2}\right)=\sqrt{\frac{1}{\# h} \sum_{f=1}^{\# h}\left(\gamma_{1}^{f}-\gamma_{2}^{f}\right)^{2}}
$$

Similar to the situations that are described and managed by HFSs [9] where the DMs may consider several possible values to define a membership function, Rodriguez et al. [7] introduced the concept of HFLTS to handle the situations in linguistic contexts where the DMs hesitant among several possible linguistic values to express their assessments.
Definition 2.3 [7]. Let $S=\left\{s_{0}, \cdots, s_{g}\right\}$ be a linguistic term set, a HFLTS $H_{S}$ is defined as an ordered finite subset of consecutive linguistic terms of $S$ :

$$
H_{s}=\left\{s^{f} \mid f=1, \cdots, \# H_{s}\right\}(s \in S)
$$

where $\# H_{s}$ is the number of all linguistic values that compound the HFLTS $H_{S}$.

Example 2.1. Let $S=\left\{s_{0}, \cdots, s_{6}\right\}$ be a linguistic term set with seven-point rating scales, so two different HFLTSs $H_{S}^{1}$ and $H_{S}^{2}$ might be as: $H_{S}^{1}=\left\{s_{1}, s_{2}\right\}, H_{S}^{2}=\left\{s_{3}, s_{4}, s_{5}\right\}$.

In the real decision process, the DMs usually employ the comparative linguistic expressions which are very close to human being's cognitive model and also provide the DMs with greater flexibility to elicit linguistic expressions, to express their assessments. Rodriguez et al. [7] proposed a transformation function $E_{G_{H}}$ that transforms the comparative linguistic expressions into HFLTS as follows:

$$
\begin{aligned}
& E_{G_{H}}\left(s_{i}\right)=\left\{s_{i} \mid s_{i} \in S\right\}, \\
& E_{G_{H}}\left(\text { at most } s_{i}\right)=\left\{s_{j} \mid s_{j} \leq s_{i} \text { and } s_{i}, s_{j} \in S\right\}, \\
& E_{G_{H}}\left(\text { at least } s_{i}\right)=\left\{s_{j} \mid s_{j} \geq s_{i} \text { and } s_{i}, s_{j} \in S\right\}, \\
& E_{G_{H}}\left(\text { between } s_{i} \text { and } s_{j}\right)=\left\{s_{k} \mid s_{i} \leq s_{k} \leq s_{j} \text { and } s_{i}, s_{k}, s_{j} \in S\right\} .
\end{aligned}
$$

Example 2.2. Let $S=\left\{s_{0}, \cdots, s_{6}\right\}$ be a linguistic term set with seven-point rating scales, $l l_{1}=\left\{\right.$ at most $\left.s_{2}\right\}$ and $l l_{2}=\left\{\right.$ between $s_{1}$ and $\left.s_{3}\right\}$ be two comparative linguistic expressions. According to the above transformation function $E_{G_{H}}$, they can be converted into two HFLTSs as follows:

$$
E_{G_{H}}\left(l l_{1}\right)=\left\{s_{0}, s_{1}, s_{2}\right\}, E_{G_{H}}\left(l l_{2}\right)=\left\{s_{1}, s_{2}, s_{3}\right\} .
$$

To effectively capture the semantics of the HFLTSs, Zhang and $\mathrm{Xu}$ [15] proposed a concept of HTrFN. The HTrFN benefited from both the superiority of the trapezoidal fuzzy number (TrFN) and the HFE, has strong ability to tackle the imprecise and ambiguous information in real-world applications, which is defined as below:

Definition 2.4 [15]. Let $X$ be a fixed set, a hesitant trapezoidal fuzzy set (HTrFS) $\tilde{A}$ on $X$ is defined as follows:

$$
\tilde{A}=\left\{<x, \tilde{h}_{\tilde{A}}(x)>\mid x \in X\right\}
$$

where $\tilde{h}_{\tilde{A}}(x)$ is a set of several TrFNs, representing some possible membership degrees of the element $x \in X$ to $\tilde{A}$. 
For convenience, $\tilde{h}_{\tilde{A}}(x)$ is called a HTrFN, denoted by $\tilde{h}=\left\{\tilde{\alpha}^{f} \mid f=1,2, \cdots, \# \tilde{h}\right\}$, where $\tilde{\alpha}_{f}=T\left({ }_{a} \gamma^{f},{ }_{b} \gamma^{f},{ }_{c} \gamma^{f},{ }_{d} \gamma^{f}\right)$ is a $\operatorname{TrFN}$ and $\# \tilde{h}$ is the number of the TrFNs in $\tilde{h}$.

Assumption 2.2 [15]. Two basic assumptions are made: (1) All possible TrFNs of the HTrFNs $\tilde{h}$ are arranged in a decreasing order, and thus $\tilde{\alpha}^{f}$ is the $f^{\text {th }}$ largest TrFN in $\tilde{h}$. (2) In order to have a correct comparison, the two corresponding HTrFNs should have the same length. For any two HTrFNs $\tilde{h}_{1}$ and $\tilde{h}_{2}$, if there are fewer TrFNs in $\tilde{h}_{1}$ than in $\tilde{h}_{2}$, an extension of $\tilde{h}_{1}$ should be considered pessimistically by repeating its minimum TrFN until it has the same length with $\tilde{h}_{2}$.

Two distance measures for HTrFNs were proposed by Zhang and $\mathrm{Xu}[15]$ as follows:

Definition 2.5 [15]. Given two HTrFNs $\tilde{h}_{i}=\left\{\tilde{\alpha}_{i}^{f}=T\left({ }_{a} \gamma_{i}^{f},{ }_{b} \gamma_{i}^{f},{ }_{c} \gamma_{i}^{f},{ }_{d} \gamma_{i}^{f}\right) \mid f=1,2, \cdots, \# \tilde{h}_{i}\right\}(i=1,2)$, and assume $\# \tilde{h}=\# \tilde{h}_{1}=\# \tilde{h}_{2}$, the hesitant trapezoidal Hamming and Euclidean distances between them can be defined, respectively, as follows:

$$
d\left(\tilde{h}_{1}, \tilde{h}_{2}\right)=\frac{1}{6 \# \tilde{h}}\left(\sum_{f=1}^{\# \tilde{h}}\left(\begin{array}{l}
\left|{ }_{a} \gamma_{1}^{f}-{ }_{a} \gamma_{2}^{f}\right|+2\left|{ }_{b} \gamma_{1}^{f}-{ }_{b} \gamma_{2}^{f}\right| \\
+2\left|{ }_{c} \gamma_{1}^{f}-{ }_{c} \gamma_{2}^{f}\right|+\left|{ }_{d} \gamma_{1}^{f}-{ }_{d} \gamma_{2}^{f}\right|
\end{array}\right)\right)
$$

and

$$
d\left(\tilde{h}_{1}, \tilde{h}_{2}\right)=\sqrt{\frac{1}{6 \# \tilde{h}\left(\sum_{f=1}^{\# \tilde{h}}\left(\begin{array}{l}
\left({ }_{a} \gamma_{1}^{f}-{ }_{a} \gamma_{2}^{f}\right)^{2}+2\left({ }_{b} \gamma_{1}^{f}-{ }_{b} \gamma_{2}^{f}\right)^{2} \\
+2\left({ }_{c} \gamma_{1}^{f}-{ }_{c} \gamma_{2}^{f}\right)^{2}+\left({ }_{d} \gamma_{1}^{f}-{ }_{d} \gamma_{2}^{f}\right)^{2}
\end{array}\right)\right)}}
$$

\section{HESITANT HETEROGENEOUS TOPSIS MULTIPLE CRITERIA ANALYSIS APPROACH}

\section{A. Hesitant heterogeneous MCDM problem}

Consider a MCDM problem under a hesitant heterogeneous environment, let $\boldsymbol{A}=\left\{A_{1}, A_{2}, \cdots, A_{m}\right\}$ $(m \geq 2)$ be a discrete set of $m$ feasible alternatives, $\boldsymbol{C}=\left\{C_{1}, C_{2}, \cdots, C_{n}\right\}$ be a finite set of criteria. The set of criteria $\boldsymbol{C}$ can be divided into two subsets, $\boldsymbol{C}_{E}$ and $\boldsymbol{C}_{F}$, representing the criteria whose values are in formats of HFEs and HFLTSs, respectively. Let $\boldsymbol{C}_{E}=\left\{C_{1}, C_{2}, \cdots, C_{e}\right\}$, $\boldsymbol{C}_{F}=\left\{C_{e+1}, C_{e+2}, \cdots, C_{n}\right\}$ where $1 \leq e \leq n$. Thus $\boldsymbol{C}_{E} \cup \boldsymbol{C}_{F}=\boldsymbol{C}$ and $\boldsymbol{C}_{E} \cap \boldsymbol{C}_{F}=\varnothing(\varnothing$ is the empty set $)$. For convenience, we denote the subscripts of these two subsets $\boldsymbol{C}_{E}$ and $\boldsymbol{C}_{F}$ as $N_{1}=\{1,2, \cdots, e\}$ and $N_{2}=\{e+1, e+2, \cdots, n\}$, respectively, and let $M=\{1,2, \cdots, m\}, N=\{1,2, \cdots, n\}$, the ratings of the alternative $A_{i}(i \in M)$ on the criteria $C_{j}(j \in N)$ be denoted by $x_{i j}$. Therefore, we obtain

$$
x_{i j}=\left\{\begin{array}{l}
h_{i j}=\left\{\gamma_{i j}^{f} \mid f=1,2, \cdots, \# h_{i j}\right\}, i \in M, j \in N_{1} \\
H_{S}^{i j}=\left\{\left(s^{f}\right)_{i j} \mid f=1, \cdots, \# H_{S}^{i j}, s \in S\right\}, i \in M, j \in N_{2}
\end{array}\right.
$$

In this paper, we employ the HTrFNs to represent the semantics of the HFLTSs. Thus the $x_{i j}\left(i \in M, j \in N_{2}\right)$ can further denoted by $\tilde{h}_{i j}=\left\{\tilde{\alpha}_{i j}^{f} \mid f=1,2, \cdots, \# \tilde{h}_{i j}\right\}$, where $\tilde{\alpha}_{i j}^{f}$ is the TrFNs denoted by $\tilde{\alpha}_{i j}^{f}=T\left({ }_{a} \gamma_{i j}^{f},{ }_{b} \gamma_{i j}^{f},{ }_{c} \gamma_{i j}^{f},{ }_{d} \gamma_{i j}^{f}\right)$ and $\# \tilde{h}_{i j}\left(=\# H_{S}^{i j}\right)$ is the number of TrFNs in $\tilde{h}_{i j}$. Thus the Eq. (3.1) can be rewritten as:

$$
x_{i j}=\left\{\begin{array}{l}
h_{i j}=\left\{\begin{array}{l}
\left.\gamma_{i j}^{f} \mid f=1,2, \cdots, \# h_{i j}\right\}, i \in M, j \in N_{1} \\
\tilde{h}_{i j}=\left\{\begin{array}{l}
\tilde{\alpha}_{i j}^{f}=T\left({ }_{a} \gamma_{i j}^{f},{ }_{b} \gamma_{i j}^{f},{ }_{c} \gamma_{i j}^{f},{ }_{d} \gamma_{i j}^{f}\right) \\
\mid f=1,2, \cdots, \# \tilde{h}_{i j}
\end{array}\right\}, i \in M, j \in N_{2}
\end{array}\right.
\end{array}\right.
$$

Therefore, the hesitant heterogeneous MCDM problem can be concisely expressed in the matrix format as below:

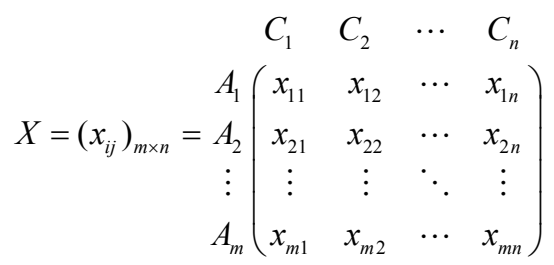

In practical decision process, the weights of criteria should be taken into account. Here we denote the criteria weighting vector by $\boldsymbol{w}=\left(w_{1}, w_{2}, \cdots, w_{n}\right)^{\mathrm{T}}$, where $w_{j}$ is the relative weight of the criterion $C_{j}(j \in N)$, satisfying the normalization condition: $\sum_{j=1}^{n} w_{j}=1$ and $w_{j} \geq 0(j \in N)$. Moreover, the criteria set $\boldsymbol{C}_{E}\left(\boldsymbol{C}_{F}\right)$ can be further divided into two sets, $\boldsymbol{C}_{E}^{B}$ and $\boldsymbol{C}_{E}^{C}\left(\boldsymbol{C}_{F}^{B}\right.$ and $\left.\boldsymbol{C}_{F}^{C}\right)$, where $\boldsymbol{C}_{E}^{B}\left(\boldsymbol{C}_{F}^{B}\right)$ represents a collection of benefit criteria (the larger the criteria values the better) and $\boldsymbol{C}_{E}^{C}\left(\boldsymbol{C}_{F}^{C}\right)$ denotes a collection of cost criteria (the smaller the criteria values the better). To eliminate the effect of different physical dimensions and measurements on the final decision results, in the decision process we transform the criteria values of the cost type into the criteria values of the benefit type by using the following equation: 


$$
x_{i j}^{N}=\left\{\begin{array}{l}
h_{h i j}\left(=\left\{\gamma_{i j}^{f} \mid f=1,2, \cdots, \# h_{i j}\right\}\right), C_{j} \in \boldsymbol{C}_{E}^{B} \\
\left(1-h_{i j}\right)\left(=\left\{\begin{array}{l}
\left.\left.\left(1-\gamma_{i j}^{f}\right) \mid f=1,2, \cdots, \# h_{i j}\right\}\right), C_{j} \in \boldsymbol{C}_{E}^{C} \\
\left({ }_{a} \gamma_{i j}^{f}, \gamma_{b i j}^{f}, \gamma_{i j}^{f},{ }_{d} \gamma_{i j}^{f}\right) \\
f=1,2, \cdots, \# \tilde{h}_{i j}
\end{array}\right\}\right), C_{j} \in \boldsymbol{C}_{F}^{B} \\
\left(1-\tilde{h}_{i j}\right)\left(=\left\{\begin{array}{l}
T\left(1-{ }_{d} \gamma_{i j}^{f}, 1-{ }_{c} \gamma_{i j}^{f}, 1-{ }_{b} \gamma_{i j}^{f}, 1-{ }_{a} \gamma_{i j}^{f}\right) \\
f=1,2, \cdots, \# \tilde{h}_{i j}
\end{array}\right\}\right), C_{j} \in \boldsymbol{C}_{F}^{C}
\end{array}\right.
$$

\section{B. The extended TOPSIS method}

We here extend the TOPSIS method to take into account hesitant heterogeneous information and utilize it to obtain the final ranking of the alternatives. TOPSIS, proposed by Hwang and Yoon [14], is a kind of method to solve MADM problems, which aims at choosing the alternative with the shortest distance from the positive ideal solution (PIS) and the farthest distance from the negative ideal solution (NIS), and is widely used for tackling the ranking problems in real situations.

The extended TOPSIS method starts with the determination of the hesitant heterogeneous PIS (HH-PIS) and the hesitant heterogeneous NIS (HH-NIS). Let $A^{+}=\left\{x_{j}^{+} \mid j \in N\right\}$ denote the HH-PIS and $A^{-}=\left\{x_{j}^{-} \mid j \in N\right\}$ denote the HH-NIS, respectively, which be defined as follows:

$$
x_{j}^{+}=\left\{\begin{array}{l}
=\left\{\gamma_{j}^{+f}=\max _{i} \gamma_{i j}^{f}(f=1,2, \cdots, \# h)\right\}, j \in N_{1} \\
=\left\{\tilde{\alpha}_{j}^{+f}=\max _{i} \tilde{\alpha}_{i j}^{f}(f=1,2, \cdots, \# h)\right\}, j \in N_{2}
\end{array}\right.
$$

and

$$
x_{j}^{-}=\left\{\begin{array}{l}
=\left\{\gamma_{j}^{-f}=\min _{i} \gamma_{i j}^{f}(f=1,2, \cdots, \# h)\right\}, j \in N_{1} \\
=\left\{\tilde{\alpha}_{j}^{-f}=\min _{i} \tilde{\alpha}_{i j}^{f}(f=1,2, \cdots, \# h)\right\}, j \in N_{2}
\end{array}\right.
$$

The separations between alternatives under each criterion are usually measured by the Euclidean distance. Thus the separation measures, $d_{i j}^{+}$and $d_{i j}^{-}$, of each alternative $A_{i}$ with respective to each criterion $C_{j}$ from the HH-PIS $A^{+}$and the HH-NIS $A^{-}$, respectively, are derived from the following formulas:

$$
d_{i j}^{+}=\left\{\begin{array}{l}
d\left(h_{i j}, h_{j}^{+}\right)=\sqrt{\frac{1}{\# h} \sum_{f=1}^{\# h}\left(\gamma_{i j}^{f}-\gamma_{j}^{+f}\right)^{2}},\left(j \in N_{1}\right) \\
d\left(\tilde{h}_{i j}, \tilde{h}_{j}^{+}\right)=\sqrt{\frac{1}{6 \# \tilde{h}}\left(\sum_{f=1}^{\# \tilde{h}}\left(\begin{array}{l}
\left.{ }_{a} \gamma_{i j}^{f}-{ }_{a} \gamma_{i j}^{+f}\right)^{2}+2\left({ }_{b} \gamma_{i j}^{f}-\gamma_{b} \gamma_{i j}^{+f}\right)^{2} \\
+2\left({ }_{c} \gamma_{i j}^{f}-\gamma_{c} \gamma_{i j}^{+f}\right)^{2}+\left({ }_{d} \gamma_{i j}^{f}-{ }_{d} \gamma_{i j}^{+f}\right)^{2}
\end{array}\right)\right),\left(j \in N_{2}\right)}
\end{array}\right.
$$

and

$$
d_{i j}^{-}=\left\{\begin{array}{l}
d\left(h_{i j}, h_{j}^{-}\right)=\sqrt{\frac{1}{\# h} \sum_{f=1}^{\# h}\left(\gamma_{i j}^{f}-\gamma_{j}^{-f}\right)^{2}},\left(j \in N_{2}\right) \\
d\left(\tilde{h}_{i j}, \tilde{h}_{j}^{-}\right)=\sqrt{\frac{1}{6 \# \tilde{h}}\left(\sum_{f=1}^{\# \tilde{h}}\left(\begin{array}{l}
\left({ }_{a} \gamma_{i j}^{f}-{ }_{a} \gamma_{i j}^{-f}\right)^{2}+2\left({ }_{b} \gamma_{i j}^{f}-{ }_{b} \gamma_{i j}^{-f}\right)^{2} \\
\left.+2{ }_{c} \gamma_{i j}^{f}-{ }_{c} \gamma_{i j}^{-f}\right)^{2}+\left({ }_{d} \gamma_{i j}^{f}-{ }_{d} \gamma_{i j}^{-f}\right)^{2}
\end{array}\right)\right)},\left(j \in N_{2}\right)
\end{array}\right.
$$

To ensure the compatibility of all criteria, we normalize the separations of each alternative $A_{i}$ with respective to each criterion $C_{j}$ from the HH-PIS $A^{+}$and the HH-NIS $A^{-}$ using the following equations, respectively:

$$
\left(d_{i j}^{+}\right)^{*}=\left\{\begin{array}{l}
=\frac{d\left(h_{i j}, h_{j}^{+}\right)}{\sum_{i=1}^{m} d\left(h_{i j}, h_{j}^{+}\right)},\left(i \in M, j \in N_{1}\right) \\
=\frac{d\left(\tilde{h}_{i j}, \tilde{h}_{j}^{+}\right)}{\sum_{i=1}^{m} d\left(\tilde{h}_{i j}, \tilde{h}_{j}^{+}\right)},\left(i \in M, j \in N_{2}\right)
\end{array}\right.
$$

and

$$
\left(d_{i j}^{-}\right)^{*}=\left\{\begin{array}{l}
=\frac{d\left(h_{i j}, h_{j}^{-}\right)}{\sum_{i=1}^{m} d\left(h_{i j}, h_{j}^{-}\right)},\left(i \in M, j \in N_{1}\right) \\
=\frac{d\left(\tilde{h}_{i j}, \tilde{h}_{j}^{-}\right)}{\sum_{i=1}^{m} d\left(\tilde{h}_{i j}, \tilde{h}_{j}^{-}\right)},\left(i \in M, j \in N_{2}\right)
\end{array}\right.
$$

Thus the weight separation measures $d_{i}^{+}$and $d_{i}^{-}$of each alternative $A_{i}$ from the HH-PIS $A^{+}$and the HH-NIS $A^{-}$can be determined respectively using the following formulas:

$$
d_{i}^{+}=\sum_{j=1}^{n} w_{j}\left(d_{i j}^{+}\right)^{*}(i \in M)
$$

and

$$
d_{i}^{-}=\sum_{j=1}^{n} w_{j}\left(d_{i j}^{-}\right)^{*}(i \in M)
$$

The relative closeness coefficient of each alternative $A_{i}$ with respect to the HH-PIS $A^{+}$is defined as the following formula:

$$
C C_{i}=\frac{d_{i}^{-}}{d_{i}^{+}+d_{i}^{-}}
$$


where $0 \leq C C_{i} \leq 1(i \in M)$.

Obviously, the alternative $A_{i}$ is closer to the HH-PIS $A^{+}$and farther from the HH-NIS $A^{-}$as the $C C_{i}$ approaches to 1 . Therefore, by comparing the closeness coefficient $C C_{i}$, we can determine the ranking order of all alternatives and select the best one from a set of feasible alternatives.

\section{The proposed algorithm}

Based on the above analysis, the algorithm of the extended TOPSIS method for solving the hesitant heterogeneous MCDM problem can be summarized as:

Step 1. Form the hesitant heterogeneous MCDM problem and identify the corresponding decision matrix.

Step 2. Determine the HH-PIS $A^{+}$and the HH-NIS $A^{-}$ by using Eqs. (3.5) and (3.6), respectively.

Step 3. Utilize Eqs. (3.7) and (3.8) to calculate the separation measures $d_{i j}^{+}$and $d_{i j}^{-}$of each alternative $A_{i}$ under each criterion $C_{j}$ from the HH-PIS $A^{+}$and the HHNIS $A^{-}$, respectively.

Step 4. Normalize the separation measures $d_{i j}^{+}$and $d_{i j}^{-}$ by using the Eqs. (3.9) and (3.10), to obtain the normalized separation measures $\left(d_{i j}^{+}\right)^{*}$ and $\left(d_{i j}^{-}\right)^{*}$, respectively.

Step 5. Calculate the weight separation measures $d_{i}^{+}$ and $d_{i}^{-}$of each alternative $A_{i}$ by using Eqs. (3.11) and (3.12), respectively.

Step 6. Compute the relative closeness coefficient $C C_{i}$ of each alternative $A_{i}$ to the HH-PIS $A^{+}$by using the Eq. (3.13).

Step 7. Rank all the alternatives $A_{i}(i \in M)$ according to the relative closeness coefficients $C C_{i}(i \in M)$ to the HH-PIS $A^{+}$and select the most desirable one(s).

\section{CASE ILlUSTRATION}

To show the usefulness of the proposed method, this section presents a MCDM problem concerned with an

automotive company which wants to select the most suitable green supplier by taking environmental performances into account.

Suppose that there are three potential green suppliers $\boldsymbol{A}=\left\{A_{1}, A_{2}, A_{3}\right\}$ are evaluated by the following four important criteria $C=\left\{C_{1}, C_{2}, C_{3}, C_{4}\right\}: C_{1}$ Product cost, $C_{2}$ Product quality, $C_{3}$ Service performance and $C_{4}$ environmental performance. The criterion $C_{1}$ is the cost criterion, and the others are the benefit criteria. The subjective important of the criteria given by the DM is $\boldsymbol{w}=\left(w_{1}, w_{2}, w_{3}, w_{4}\right)^{T}=(0.2,0.3,0.2,0.3)^{T}$. Because the increasing complexity of the socio-economic context and the vagueness of inherent subjective nature of human think, it is difficult for the decision maker of the company to provide their assessments of all alternatives by means of crisp values in qualitative criteria and single linguistic terms for the qualitative criteria. Thus the decision maker may employ the HFEs and the comparative linguistic expressions based on HFLTSs to express the assessments for the three potential suppliers. The evaluation results are listed in Table I. Linguistic terms and their corresponding $\mathrm{TrFNs}$ are described in Table II.

TABLE I. THE HESITANT HETEROGENEOUS DECISION MATRIX

\begin{tabular}{lllll}
\hline & $\mathrm{C}_{1}$ & $\mathrm{C}_{2}$ & $\mathrm{C}_{3}$ & $\mathrm{C}_{4}$ \\
\hline $\mathrm{A}_{1}$ & $\{0.7,0.5\}$ & $\begin{array}{l}\text { Between } \mathrm{MG} \\
\text { and } \mathrm{G}\end{array}$ & $\begin{array}{l}\text { Between MP } \\
\text { and MG }\end{array}$ & $\mathrm{G}$ \\
\hline $\mathrm{A}_{2}$ & $\{0.6,0.5,0.2\}$ & At least $\mathrm{G}$ & $\mathrm{F}$ & $\begin{array}{l}\text { Between } \mathrm{F} \\
\text { and G }\end{array}$ \\
\hline $\mathrm{A}_{3}$ & $\{0.5,0.3\}$ & $\mathrm{G}$ & $\begin{array}{l}\text { Between F and } \\
\mathrm{MG}\end{array}$ & At least MG \\
\hline
\end{tabular}

TABLE II. LINGUISTIC TERMS AND THE CORRESPONDING TRFNS

\begin{tabular}{lll}
\hline Linguistic terms & Abbreviation & TrFNs \\
\hline $\mathrm{s}_{0}:$ Very poor & VP & $\mathrm{T}(0.0,0.0,0.1,0.2)$ \\
\hline $\mathrm{s}_{1}:$ Poor & $\mathrm{P}$ & $\mathrm{T}(0.1,0.2,0.2,0.3)$ \\
\hline $\mathrm{s}_{2}:$ Medium poor & $\mathrm{MP}$ & $\mathrm{T}(0.2,0.3,0.4,0.5)$ \\
\hline $\mathrm{s}_{3}:$ Fair & $\mathrm{F}$ & $\mathrm{T}(0.4,0.5,0.5,0.6)$ \\
\hline $\mathrm{s}_{4}:$ Medium good & $\mathrm{MG}$ & $\mathrm{T}(0.5,0.6,0.7,0.8)$ \\
\hline $\mathrm{s}_{5}:$ Good & $\mathrm{G}$ & $\mathrm{T}(0.7,0.8,0.8,0.9)$ \\
\hline $\mathrm{s}_{6}:$ Very good & $\mathrm{VG}$ & $\mathrm{T}(0.8,0.9,1.0,1.0)$ \\
\hline
\end{tabular}

TABLE III.

THE NORMALIZED HESITANT HETEROGENEOUS DECISION MATRIX

\begin{tabular}{|c|c|c|c|c|}
\hline & $\mathrm{C}_{1}$ & $\mathrm{C}_{2}$ & $\mathrm{C}_{3}$ & $\mathrm{C}_{4}$ \\
\hline$A_{1}$ & $\{0.5,0.3,0.3\}$ & $\begin{array}{l}\{\mathrm{T}(0.7,0.8,0.8,0.9) \\
\mathrm{T}(0.5,0.6,0.7,0.8)\}\end{array}$ & $\begin{array}{l}\{\mathrm{T}(0.5,0.6,0.7,0.8), \\
\mathrm{T}(0.4,0.5,0.5,0.6), \\
\mathrm{T}(0.2,0.3,0.4,0.5)\}\end{array}$ & $\begin{array}{l}\{\mathrm{T}(0.7,0.8,0.8,0.9), \\
\mathrm{T}(0.7,0.8,0.8,0.9), \\
\mathrm{T}(0.7,0.8,0.8,0.9)\}\end{array}$ \\
\hline$A_{2}$ & $\{0.8,0.5,0.4\}$ & $\begin{array}{l}\{\mathrm{T}(0.8,0.9,1.0,1.0) \\
\mathrm{T}(0.7,0.8,0.8,0.9)\}\end{array}$ & $\begin{array}{l}\{\mathrm{T}(0.4,0.5,0.5,0.6), \\
\mathrm{T}(0.4,0.5,0.5,0.6), \\
\mathrm{T}(0.4,0.5,0.5,0.6)\}\end{array}$ & $\begin{array}{l}\{\mathrm{T}(0.7,0.8,0.8,0.9), \\
\mathrm{T}(0.5,0.6,0.7,0.8), \\
\mathrm{T}(0.4,0.5,0.5,0.6)\}\end{array}$ \\
\hline$A_{3}$ & $\{0.7,0.5,0.5\}$ & $\begin{array}{l}\{\mathrm{T}(0.7,0.8,0.8,0.9) \\
\mathrm{T}(0.7,0.8,0.8,0.9)\}\end{array}$ & $\begin{array}{l}\{\mathrm{T}(0.5,0.6,0.7,0.8), \\
\mathrm{T}(0.4,0.5,0.5,0.6), \\
\mathrm{T}(0.4,0.5,0.5,0.6)\}\end{array}$ & $\begin{array}{l}\{\mathrm{T}(0.8,0.9,1.0,1.0), \\
\mathrm{T}(0.7,0.8,0.8,0.9), \\
\mathrm{T}(0.5,0.6,0.7,0.8)\}\end{array}$ \\
\hline$A^{+}$ & $\{0.8,0.5,0.5\}$ & $\begin{array}{l}\{\mathrm{T}(0.8,0.9,1.0,1.0) \\
\mathrm{T}(0.7,0.8,0.8,0.9)\}\end{array}$ & $\begin{array}{l}\{\mathrm{T}(0.5,0.6,0.7,0.8), \\
\mathrm{T}(0.4,0.5,0.5,0.6), \\
\mathrm{T}(0.4,0.5,0.5,0.6)\}\end{array}$ & $\begin{array}{l}\{\mathrm{T}(0.8,0.9,1.0,1.0), \\
\mathrm{T}(0.7,0.8,0.8,0.9), \\
\mathrm{T}(0.7,0.8,0.8,0.9),\}\end{array}$ \\
\hline$A^{-}$ & $\{0.5,0.3,0.3\}$ & $\begin{array}{l}\{\mathrm{T}(0.7,0.8,0.8,0.9) \\
\mathrm{T}(0.5,0.6,0.7,0.8)\}\end{array}$ & $\begin{array}{l}\{\mathrm{T}(0.4,0.5,0.5,0.6), \\
\mathrm{T}(0.4,0.5,0.5,0.6), \\
\mathrm{T}(0.2,0.3,0.4,0.5)\}\end{array}$ & $\begin{array}{l}\{\mathrm{T}(0.7,0.8,0.8,0.9), \\
\mathrm{T}(0.5,0.6,0.7,0.8), \\
\mathrm{T}(0.4,0.5,0.5,0.6)\}\end{array}$ \\
\hline
\end{tabular}


TABLE IV. THE CLOSENESS COEFFICIENTS OF ALTERNATIVES ALONG WITH THEIR FINAL RANKING

\begin{tabular}{lllll}
\hline & $d_{i}^{+}$ & $d_{i}^{-}$ & $C C_{i}$ & Ranking \\
\hline$A_{1}$ & 0.4783 & 0.2271 & 0.3219 & 3 \\
\hline$A_{2}$ & 0.2979 & 0.3343 & 0.5288 & 2 \\
\hline$A_{3}$ & 0.2238 & 0.4407 & 0.6632 & 1 \\
\hline
\end{tabular}

In the following, we employ the proposed method to aid the automotive company select the most suitable green supplier. The solution process and the computation results are summarized as follows:

Firstly, we convert the HFLTSs into the HTrFNs and employ the Eq. (3.4) to transform criteria values of the cost type into the criteria values of the benefit type, the results are listed in Table III. Secondly, we utilize the Eqs.(3.5) and (3.6) to determine the corresponding HH-PIS $A^{+}$and the HH-NIS $A^{-}$as in Table III. At length, we employ the Eqs. (3.7)-(3.13) to calculate the corresponding separation measures $d_{i}^{+}$and $d_{i}^{-}$, and the relative closeness coefficient $C C_{i}(i=1,2,3)$, respectively. The results are presented in Table IV, together with the corresponding rankings on the basis of $C C_{i}$.

It is easy to see that the optimal order for these three potential suppliers is $A_{3} \succ A_{2} \succ A_{1}$, and thus the supplier $\left(A_{1}\right)$ is the most desirable supplier. Obviously, the proposed method is not only simple and easy to understand but also reduces the loss of the original data information by using the unified methods proposed by Rodriguez et al. [8].

\section{CONCLUSIONS}

TOPSIS method is one of the well-known methods for solving the MCDM problem. This paper proposes an extend TOPSIS method to handle the hesitant heterogeneous MCDM problem in which the ratings of alternatives take the different formats such as HFEs and HFLTSs. In the proposed method, the semantics of the HFLTSs are represented by the HTrFNs. Based on different distance measures, the separations to the ideal solution and negative ideal solution under each criterion are calculated, respectively. Then the separations under each criterion are normalized in order to ensure the compatibility of all criteria. Finally, the optimal alternative which is closest to the ideal solution and remotest from negative ideal solution is identified. The proposed method is not only very simple and easy to understand but also reduces the loss of the original data information by using the unified methods proposed by Rodriguez et al. [8]. Thus, the proposed approach provides us an effective and practical way to handle the hesitant heterogeneous MCDM problem in case of considering the DM's hesitation.

\section{ACKNOWLEDGMENT}

The authors are very grateful to the anonymous reviewers and the editor for their insightful and constructive comments and suggestions that have led to an improved version of this paper. The work was supported by the National Natural Science Foundation of China (No.61273209), the Fundamental Research Funds for the Central Universities (No.CXZZ13 0139) and the Scientific Research Foundation of Graduate School of Southeast University (No. YBJJ1339).

\section{REFERENCES}

[1] S.-J. Chuu, Selecting the advanced manufacturing technology using fuzzy multiple attributes group decision making with multiple fuzzy information, Computers \& industrial engineering, 57 (2009) 10331042 .

[2] B. Farhadinia, Information measures for hesitant fuzzy sets and interval-valued hesitant fuzzy sets, Information Sciences, 240 (2013) 129-144.

[3] F. Herrera, L. Martínez, A 2-tuple fuzzy linguistic representation model for computing with words, Fuzzy Systems, IEEE Transactions on, 8 (2000) 746-752.

[4] F. Herrera, L. Martınez, P.J. Sánchez, Managing non-homogeneous information in group decision making, European Journal of Operational Research, 166 (2005) 115-132.

[5] D.-F. Li, Z.-G. Huang, G.-H. Chen, A systematic approach to heterogeneous multiattribute group decision making, Computers \& Industrial Engineering, 59 (2010) 561-572.

[6] L. Martínez, J. Liu, D. Ruan, J.-B. Yang, Dealing with heterogeneous information in engineering evaluation processes, Information Sciences, 177 (2007) 1533-1542.

[7] R.M. Rodriguez, L. Martinez, F. Herrera, Hesitant fuzzy linguistic term sets for decision making, Fuzzy Systems, IEEE Transactions on, 20 (2012) 109-119.

[8] R.M. Rodrıguez, P.J. Sánchez, L. Martınez, Managing hesitant heterogeneous information in decision making, Proceedings of the 2013 International Conference on Machine Learning and Cybernetics, Tianjin, 2014-2017 July, 2013.

[9] V. Torra, Hesitant fuzzy sets, International Journal of Intelligent Systems, 25 (2010) 529-539.

[10] M.M. Xia, Z.S. Xu, Hesitant fuzzy information aggregation in decision making, International journal of approximate reasoning, 52 (2011) 395-407.

[11] Z.S. Xu, M.M. Xia, Distance and similarity measures for hesitant fuzzy sets, Information Sciences, 181 (2011) 2128-2138.

[12] Z.S. Xu, M.M. Xia, On distance and correlation measures of hesitant fuzzy information, International Journal of Intelligent Systems, 26 (2011) 410-425.

[13] Z.S. Xu, X.L. Zhang, Hesitant fuzzy multi-attribute decision making based on TOPSIS with incomplete weight information, KnowledgeBased Systems, 52 (2013) 53-64.

[14] C. Hwang, K. Yoon, Multiple Attibute Decision Methods and Applications, in, Springer, Berlin Heidelberg, 1981.

[15] X.L. Zhang, Z.S. Xu, A hierarchical fuzzy QUALIFLEX method dealing with comparative linguistic expressions based on hesitant trapezoidal fuzzy numbers and applications to the evaluation of green supply chain initiatives, (2013) Technical report.

[16] X.L. Zhang, Z.S. Xu, Hesitant fuzzy agglomerative hierarchical clustering algorithms, International Journal of Systems Science, http://dx.doi.10.1080/00207721.00202013.00797037.

[17] X.L. Zhang, Z.S. Xu, An MST cluster analysis method under hesitant fuzzy environment, Control and Cybernetics 41 (2012), 645-666.

[18] X.L. Zhang, Z.S. Xu, The TODIM analysis approach based on novel measured functions under hesitant fuzzy environment, KnowledgeBased Systems, 61(2014) 48-58. 